\title{
PERIBULBAR ANAESTHESIA: FAILURE TO ABOLISH THE OCULOCARDIAC REFLEX
}

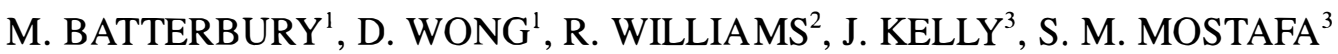 \\ Liverpool
}

\begin{abstract}
SUMMARY
Peribulbar anaesthesia is a form of regional anaesthesia which has been used with increasing popularity in cataract surgery. Recently indications for its use have been extended to include surgery for vitreo-retinal disease and strabismus. This study shows that in nine of 11 patients in whom the oculocardiac reflex was stimulated by traction on an extra-ocular muscle, peribulbar anaesthesia failed to abolish the reflex. In each of these patients, the reflex manifested as a bradycardia. In one subject, the bradycardia was profound, showing no QRS complex for five seconds. We recommend that cardiac rhythm be monitored throughout procedures performed under peribulbar anaesthesia, and that a vagolytic agent be available for immediate use.
\end{abstract}

The oculocardiac reflex is a trigemino-vagal reflex which results in cardiac arrhythmias, the most common of which is a bradycardia, in response to many stimuli, including traction on an extra-ocular muscle. ${ }^{1,2}$ Administration of atropine or glycopyrrolate may block the reflex. ${ }^{1}$

Regional anaesthesia is used frequently as an alternative to general anaesthesia in ophthalmic surgery, particularly for anterior segment surgery, but also for strabismus and more recently vitreo-retinal surgery. Retrobulbar anaesthesia is associated with uncommon but significant complications, such as orbital haemorrhage, optic nerve trauma and ocular perforation, ${ }^{3,4}$ and respiratory arrest. ${ }^{5}$ Peribulbar anaesthesia is safer but is equally effective, ${ }^{3,6}$ and has therefore come to be used with increasing frequency as an alternative to retrobulbar anaesthesia, particularly for cataract surgery. A form of peribulbar injection technique is now also used for vitreoretinal procedures performed under local anaesthesia.

It has been our practice to administer a peribulbar injection of lignocaine to certain patients undergoing vitreo-

From 'St Paul's Eye Hospital, Old Hall Street, Liverpool; ${ }^{2}$ Department of Clinical Engineering, Duncan Building, Royal Liverpool University Hospital, Liverpool; ${ }^{3}$ Department of Anaesthesia, Royal Liverpool University Hospital, Liverpool.

Correspondence to: M. Batterbury, Manchester Royal Eye Hospital, Oxford Road, Manchester. retinal surgery under general anaesthetic to prevent the oculocardiac reflex. The injection is administered to those patients for whom the reflex bradycardia would be particularly dangerous and in whom prophylactic atropine or glycopyrrolate may induce further undesirable arrhythmias. We have also found that the injection aids surgery by enhancing exposure of the globe by displacement of orbital contents.

However, we have also noted that the peribulbar injection does not always prevent the oculocardiac reflex. This study was conducted to determine whether peribulbar anaesthesia abolishes the oculocardiac reflex.

\section{METHOD}

The technique of peribulbar anaesthesia was a modification of that described elsewhere. ${ }^{3,6}$ Eight to $10 \mathrm{mls}$ of $2 \%$ plain lignocaine were deposited in and behind the orbicularis oculi muscle, at the equator of the globe and at the posterior part of the orbit, reached via inferotemporal and superonasal routes. Injections were performed without hyaluronidase, which was not available to us, before induction of general anaesthesia and approximately 30 minutes before surgery was started.

General anaesthesia was induced and maintained by a standardised technique. After peritomy, the medial rectus muscle was located and lassoed with a suture, avoiding undue traction on the muscle. The suture was hooked to an electronic force gauge with a maximum load of $1 \mathrm{~kg}$ measuring increments of $1 \mathrm{~g}$. The gauge was held by the surgeon (DW) who applied a square wave stimulus of known force. The square wave stimulus is a sudden pull on the muscle, maintained for a minimum of 20 seconds, followed by an equally sudden release. This stimulus is known to be the most potent stimulant of the reflex, ${ }^{2}$ and is similar to the forceful way in which extra-ocular muscles are manipulated during strabismus and vitreo-retinal surgery. Before and during application of the stimulus, cardiac rhythm was monitored and recorded.

The reflex was defined as positive if a decrease in heart rate of more than seven beats per minute occurred. 
The study was approved by the Ethics Committee. Patients admitted for vitreo-retinal surgery were recruited and gave written informed consent of their participation.

\section{RESULTS}

Two stimuli were used, namely $150 \mathrm{~g}$ and $500 \mathrm{~g}$ of traction. In five patients, $500 \mathrm{~g}$ was applied, and in each of these subjects the oculocardiac reflex was evident (Table I). In six patients a force of $150 \mathrm{~g}$ was applied (Table II), causing the reflex to occur in four subjects. In two patients there was no change in cardiac rhythm.

The reflex in each subject in whom it was evident was a decrease in heart rate of more than seven beats per minute. In one patient the bradycardia was so profound that no electrical complex was recorded for over four seconds.

\section{DISCUSSION}

The oculocardiac reflex is an important cause of morbidity during ophthalmic surgery, of particularly high incidence during strabismus surgery. ${ }^{1,2}$ It is a trigemino-vagal reflex which results in cardiac arrhythmias, the most common of which is a bradycardia, in response to certain stimuli. ${ }^{1,2}$ The afferent pathway is the ophthalmic division of the trigeminal nerve. The efferent pathway is the vagus nerve. Many stimuli can evoke the reflex, including traction on extra-ocular muscles and pressure on the globe. The reflex may be blocked by intramuscular or intravenous administration of atropine or glycopyrrolate, ${ }^{1}$ and may even be diminished by modifying the afferent pathway by retrobulbar anaesthesia, though this form of regional anaesthesia may itself stimulate the oculocardiac reflex. ${ }^{\text {? }}$

Peribulbar anaesthesia is a popular alternative to retrobulbar anaesthesia, particularly for cataract surgery, as it is easy to administer, safe and effective. ${ }^{3,6}$ A similar injection technique has been used for vitreo-retinal surgery without general anaesthetic.

We have been using a peribulbar injection technique during general anaesthesia in the belief that it would abolish or diminish the oculocardiac reflex. This study was set up to test this assumption, using traction on the medial rectus muscle as the applied stimulus. In our 11 subjects, a decrease in heart rate of at least 12 beats per minute occurred in eight patients, and in another patient a decrease of eight beats per minute was recorded (a fall of $16 \%)$. In only two patients was the reflex abolished.

There is no standard definition of the oculocardiac reflex, previous authors using decreases of seven or 10

Table I Cardiac rate on application of tractional force of $500 \mathrm{~g}$

\begin{tabular}{|c|c|c|c|c|}
\hline \multirow[b]{2}{*}{ Subject } & \multicolumn{2}{|c|}{ Cardiac rate ${ }^{1}$} & \multirow[b]{2}{*}{ Decrease $^{\prime}$} & \multirow[b]{2}{*}{$\%$ decrease } \\
\hline & Pre-stimulus & Post-stimulus & & \\
\hline 1 & 48 & 40 & 8 & 16 \\
\hline 2 & 58 & 27 & 31 & 53 \\
\hline 3 & 71 & 40 & 31 & 44 \\
\hline 4 & 68 & 27 & 41 & 60 \\
\hline 5 & 67 & 0 & 67 & 100 \\
\hline
\end{tabular}

'QRS complexes per minute. beats per minute or decreases of 10 or $20 \%$. $^{1}$ Using even the most strict definition, the reflex occurred in eight of 11 subjects.

There are several reasons why our peribulbar anaesthesia might not completely block the reflex. Our technique might not have been adequate, particularly as we did not use hyaluronidase, which was not available. However, we allowed 30 minutes between injection and surgery for onset of anaesthesia, which should be a sufficient interval for complete anaesthesia to occur in over $90 \%$ of cases. ${ }^{3}$ In addition, a recent study using contrast medium without hyaluronidase injected by a peribulbar technique reported that contrast medium was detectable within the muscle cone within two to six minutes and above the superior rectus muscle within 1.5 to three minutes following injection in the inferior peribulbar area. ${ }^{8}$ We believe therefore that our technique was satisfactory.

We chose the $150 \mathrm{~g}$ traction force since a force of 150 $300 \mathrm{~g}$ has previously been shown to induce the reflex in over $85 \%$ of cases in which the stimulus was applied as a square wave, the most potent of tractional stimuli. ${ }^{2}$ The $500 \mathrm{~g}$ square wave stimulus more nearly simulates traction of an extra-ocular muscle during strabismus or vitreoretinal surgery. Thus we believe our stimulus to have been adequate.

Whilst the afferent pathway for the oculocardiac reflex is known to be the trigeminal nerve, it is not certain what type of nerve fibre is involved. Candidates are pain fibres and proprioceptive fibres from the extra-ocular muscles. There are two types of pain fibre, small myelinated fast conduction fibres of maximum diameter five microns and small unmyelinated slow conduction fibres of maximum diameter 1.2 microns. Proprioceptive fibres may come from the muscle tendon (maximum diameter 12 microns) or muscle spindle (maximum diameter 20 microns). These fibres are of comparable size to the efferent motor nerves to the extra-ocular muscles, also of maximum diameter 20 microns. It is unlikely that our injection technique failed to block the small pain fibres given the known success of peribulbar anaesthesia for producing analgesia. It is also very unlikely that the injections failed to block the large diameter proprioceptive fibres when they are able to block function of the similarly sized motor fibres, producing akinesia.

It is known that the oculocardiac reflex can be induced by orbital surgery some years following enucleation. ${ }^{9}$ It is possible that peribulbar injection may not anaesthetise all

Table II Cardiac rate on application of tractional force of $150 \mathrm{~g}$

\begin{tabular}{|c|c|c|c|c|}
\hline \multirow[b]{2}{*}{ Subject } & \multicolumn{2}{|c|}{ Cardiac rate } & \multirow[b]{2}{*}{ Decrease $^{\prime}$} & \multirow[b]{2}{*}{$\%$ decrease } \\
\hline & Pre-stimulus & Post-stimulus & & \\
\hline 1 & 61 & 48 & 13 & 21 \\
\hline 2 & 67 & 43 & 24 & 35 \\
\hline 3 & 67 & 65 & 2 & 3 \\
\hline 4 & 68 & 68 & 0 & 0 \\
\hline 5 & 96 & 52 & 44 & 46 \\
\hline 6 & 72 & 36 & 36 & 50 \\
\hline
\end{tabular}

${ }^{\mathrm{I}} \mathrm{QRS}$ complexes per minute. 
trigeminal fibres in the orbit and that a positive reflex on extraocular muscle traction may occur because of transmitted traction to the origin of the muscle at the posterior orbit.

Even if abolition cannot always be achieved, some diminution of the reflex is a desirable effect, especially in the elderly patient with an unstable cardiovascular status susceptible to reduced coronary and cerebral blood flow. A controlled study is necessary to clarify fully the effects of peribulbar anaesthesia on the reflex, in particular to differentiate between abolition and diminution of the reflex.

\section{CONCLUSION}

Our study has demonstrated that the oculocardiac reflex can occur despite a peribulbar injection of lignocaine. We therefore recommend that even in the presence of successful akinesia and analgesia following peribulbar injection of anaesthetic that cardiac rhythm should be monitored throughout any procedure known to stimulate the oculocardiac reflex. A syringe charged with atropine should be available for immediate use in patients developing a significant bradycardia. We believe that monitoring is best performed by an anaesthetist who should be in attendance in case there is a need for cardiopulmonary resuscitation. We therefore stress that peribulbar anaesthesia should not be introduced as an alternative to providing trained anaesthetic staff. Finally, we also recommend that traction on an extraocular muscle be gentle and gradual, avoiding the square wave stimulus used in this study.

Salter Industrial Measurement Limited kindly provided the electronic force gauge.

Key words: Oculocardiac reflex, peribulbar anaesthesia.

\section{REFERENCES}

1. Yamashita M: Oculocardiac reflex and the anaesthesiologist. M E J Anesth 1986, 8: 399-415.

2. Blanc VF, Hardy J-F, Milot J, Jacob J-L: The oculocardiac reflex: a graphic and statistical analysis in infants and children. Can Anaesth Soc J 1983, 30: 360-9.

3. Davis DB and Mandel MR: Posterior peribulbar anaesthesia: an alternative to retrobulbar anaesthesia. $J$ Cataract Refract Surg 1986, 12: 182-4.

4. Hay A, Flynn-HW, Hoffman JI, Rivera AH: Needle penetration of the globe during retrobulbar and peribulbar injections. Ophthalmology 1991, 98: 1017-24.

5. Wittpenn JR, Rapoza P, Sternberg P, Kuwashima L, Saklad J, Patz A: Respiratory arrest following retrobulbar anaesthesia. Ophthalmology 1986, 93: 867-70.

6. Murdoch IE: Peribulbar versus retrobulbar anaesthesia. Eye 1990, 4: 445-9.

7. Pontinen PJ: The importance of the oculocardiac reflex during ocular surgery. Acta Ophthalmol (Supplement) 1966, 86: $7-66$

8. Ropo A, Nikki P, Ruusuvaara P, Kivisaari L: Comparison of retrobulbar and periocular injections of lignocaine by computerised tomography. Br J Ophthalmol 1991, 75: 417-20.

9. Kerr WJ and Vance JP: Oculocardiac reflex from the empty orbit. Anaesthesia 1983, 38: 883-5. 